\title{
Conditions of desire for psycho-oncological support services in distressed cancer patients. How can we optimize psycho-oncological support services?
}

T. Pichler ${ }^{1}$, A. Dinkel ${ }^{2}$, K. Hermelink ${ }^{3}$, P. Heußner ${ }^{4}$, B. Marten-Mittag², E. Telzerow ${ }^{4}$, P. Herschbach ${ }^{5}$ et al.

${ }^{1}$ Comprehensive Cancer Center, Munich, Germany; ${ }^{2}$ Department of Psychosomatic Medicine and Psychotherapy, Technical University of Munich, Munich, Germany; ${ }^{3}$ Clinic und Policlinic of Gynaecology, University Hospital Munich, Munich, Germany; ${ }^{4}$ Medical Clinic and Polyclinic III, University Hospital Munich, Munich, Germany; ${ }^{5}$ Roman Herzog Comprehensive Cancer Center, Department of Psychosomatic Medicine and

Psychotherapy, Technical University of Munich, Munich, Germany

\section{Introduction}

About 30 to $50 \%$ of all cancer patients are so severely distressed that they need professional psycho-oncological support. However, if such support is offered to these patients, about $60-80 \%$ refuse help.

\section{Objectives}

The aim of this study conducted by the Comprehensive Cancer Center is to identify sociodemographic, medical and psychological conditions of the desire for psychooncological support among patients with high psychosocial distress.

\section{Methods}

In a multicenter study in Germany a total of $\mathrm{n}=972$ inpatients with various cancer diagnoses were examined. Measuring instruments for psychological distress were the Distress Thermometer [1] and the QSC-R10 [2]. Further, we examined potential aspects regarding the refusal of / desire for psycho-oncological support services including sociodemographic, medical and psychological conditions (e.g. depression [3]), personality [4], social support, self-efficacy [5], previous experiences with psychological support offers and status of information.

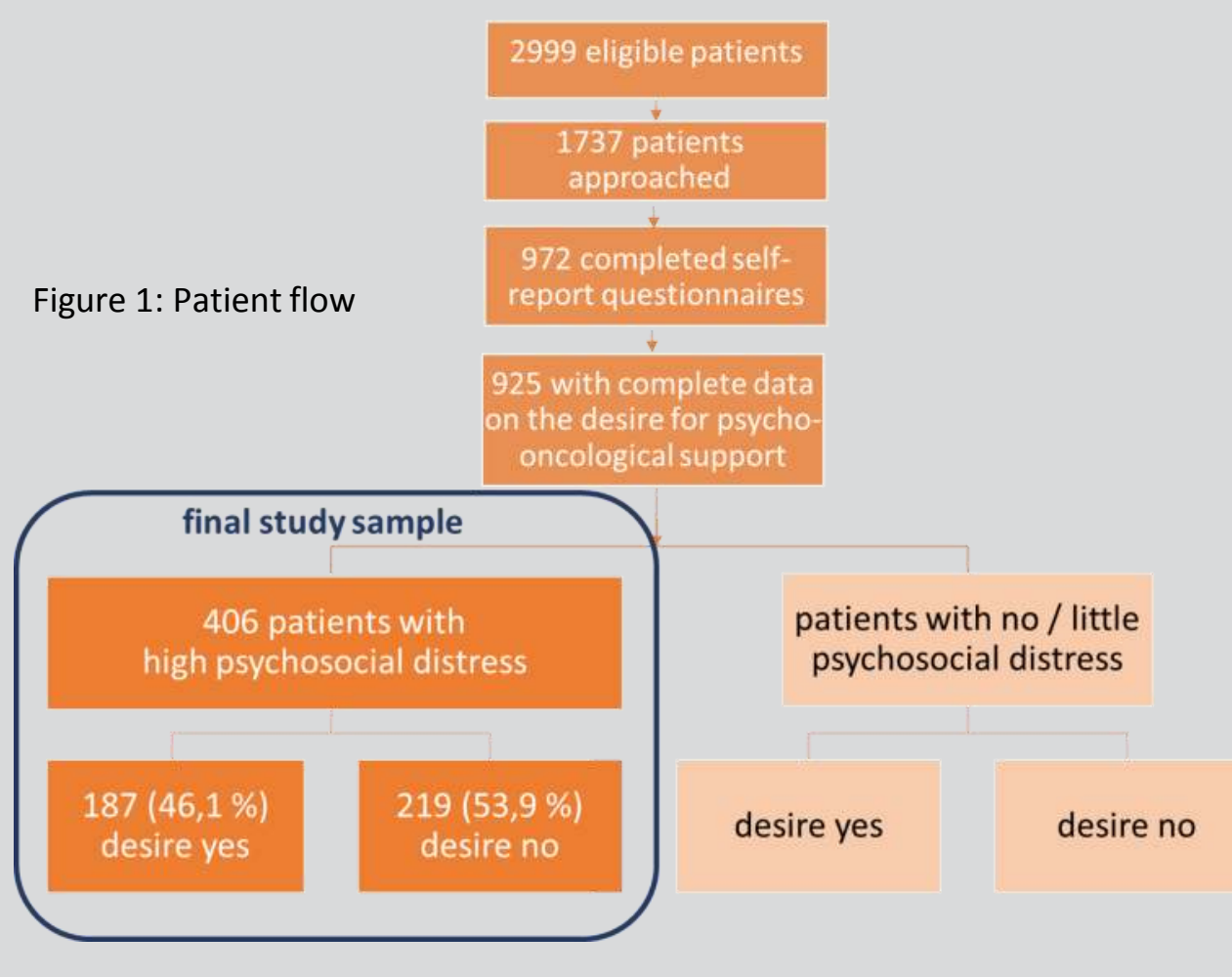

Results

Table 1: Sociodemographic sample characteristics $(N=406)$

\begin{tabular}{|c|c|c|c|c|c|}
\hline & & & Desire f & sup & \\
\hline & $\mathbf{M}$ & SD & M & SD & \\
\hline Age & 60,2 & 12,3 & 58,95 & 12,3 & .056 \\
\hline & n & $\%$ & n & $\%$ & $p$ \\
\hline Total sample & 406 & 100 & 187 & 46,1 & \\
\hline Sex & & & & & \\
\hline female & 221 & 54,4 & 128 & 57,9 & \\
\hline male & 185 & 45,6 & 59 & 31,9 & .000 \\
\hline Age group & & & & & \\
\hline 18 to 50 & 85 & 21 & 46 & 54,1 & \\
\hline 51 to 65 & 171 & 42,2 & 78 & 45,6 & \\
\hline 66 to 7 & 113 & 27,9 & 52 & 46 & \\
\hline 76 to 99 & 36 & 8,9 & 11 & 30,6 & .126 \\
\hline Cohabitating & & & & & \\
\hline yes & 270 & 66,8 & 119 & 44,1 & \\
\hline no & 134 & 33,2 & 66 & 49,3 & .325 \\
\hline
\end{tabular}

Note: $P$ values based on Chi2-tests and t-tests respectively.

- Overall more than half of highly distressed cancer patients refuse psychooncological support.

- Women wish for psycho-oncological support nearly twice as often as men. - Distressed patients who have a desire for psycho-oncological support are more depressed and have a worse physical condition than patients who refuse help. - Personality factors (neuroticism and agreeableness) and current medical treatment (chemo- and radiotherapy) do play a role for the desire for psycho-oncological support.

\section{References}

[1] Mehnert A, Müller

(2) on Distress in Cancer Patients-short form (QSC-R10) in a German sample. Psycho-Oncology 2011;20(3):287-293.

Löwe B, Kroenke K, Gräfe K. Detecting and monitoring depression with a 2-item questionnaire (PHQ 2) J J Psychosom Res. 2005;58:163-171. [4] Rammstedt B. \& John OP. Measuring personality in one minute or less: A 10-item short version of the Big Five Inventory in English and Q [5] Beierlein C, Kemper CJ, Kovaleva A, Rammstedt, B. Ein Messinstrument zur Erfassung subjektiver Kompetenzerwartungen - Allgemei
Selbstwirksamkeit Kurzskala (ASKU). GESIS Working Papers 2012;17.
Table 2: Medical sample characteristics ( $N=406)$

\begin{tabular}{|c|c|c|c|c|c|}
\hline & \multicolumn{2}{|c|}{ Total sample } & \multicolumn{3}{|c|}{ Desire for psych. support } \\
\hline & $\mathbf{n}$ & $\%$ & $\mathbf{n}$ & $\%$ & $p$ \\
\hline \multicolumn{6}{|l|}{ Tumor entity } \\
\hline Brain & 14 & 3,5 & 7 & 50 & \\
\hline Head and Neck & 30 & 7,4 & 14 & 46,7 & \\
\hline Gastrointestinal & 12 & 3 & 6 & 50 & \\
\hline Breast & 67 & 16,6 & 33 & 49,3 & \\
\hline Uterus & 26 & 6,4 & 18 & 69,2 & \\
\hline Ovaries & 29 & 7,2 & 16 & 55,2 & \\
\hline Kidney / urinary passages & 22 & 5,4 & 8 & 36,4 & \\
\hline Prostata & 85 & 21 & 21 & 24,7 & \\
\hline Bladder & 28 & 6,9 & 11 & 39,3 & \\
\hline Lung & 18 & 4,5 & 11 & 61,1 & \\
\hline Others & 28 & 6,9 & 17 & 60,7 & \\
\hline Several diagnosis & 45 & 11,1 & 25 & 55,6 & .002 \\
\hline \multicolumn{6}{|l|}{ Metastases } \\
\hline Yes & 151 & 37,9 & 71 & 47 & \\
\hline No & 165 & 41,5 & 78 & 47,3 & \\
\hline Don't know & 82 & 20,6 & 37 & 45,1 & .947 \\
\hline \multicolumn{6}{|l|}{ Duration of illness } \\
\hline Up to 3 month & 189 & 47,4 & 92 & 48,7 & \\
\hline More than 3 months & 210 & 52,6 & 94 & 44,8 & .434 \\
\hline \multicolumn{6}{|c|}{ Current medical treatment* } \\
\hline Chemotherapy & 111 & 27,3 & 63 & 56,8 & .008 \\
\hline Radiotherapy & 159 & 39,2 & 83 & 52,2 & .046 \\
\hline Hormonal therapy & 36 & 8,9 & 17 & 47,2 & .883 \\
\hline Surgery & 240 & 59,1 & 103 & 42,9 & .127 \\
\hline No treatment & 18 & 4,4 & 10 & 55,6 & .408 \\
\hline
\end{tabular}

Note: $P$ values based on Chi $^{2}$-tests and $\mathrm{t}$-tests respectively. ${ }^{*}$ One patient may receive more than one current treatment.

Table 3: Group differences between distressed patients with and without desire for psychooncological support $(N=406)$.

\begin{tabular}{|c|c|c|c|c|c|}
\hline & \multicolumn{2}{|c|}{ Total sample } & \multicolumn{3}{|c|}{ Desire for psych. support } \\
\hline & n & $\%$ & $\mathbf{n}$ & $\%$ & $p$ \\
\hline \multicolumn{6}{|c|}{ Information about psychological support offers } \\
\hline Yes & 256 & 64,2 & 112 & 43,8 & \\
\hline No & 143 & 35,8 & 73 & 51 & .161 \\
\hline \multicolumn{6}{|c|}{ Previous contact to a psychologist } \\
\hline Yes & 153 & 38 & 92 & 60,1 & \\
\hline No & 250 & 62 & 92 & 36,8 & .000 \\
\hline \multicolumn{6}{|c|}{ Social support from family / friends } \\
\hline Yes & 369 & 91,1 & 168 & 45,5 & \\
\hline \multirow[t]{2}{*}{ No } & 36 & 8,9 & 19 & 52,8 & .405 \\
\hline & M & SD & M & SD & $p$ \\
\hline Physical condition & 5,55 & 1,84 & 5,79 & 1,84 & .018 \\
\hline Depression (PHQ2) & 4,5 & 1,6 & 4,81 & 1,63 & .000 \\
\hline Self-efficacy (ASKU) & 3,85 & 0,77 & 3,79 & 0,81 & .104 \\
\hline \multicolumn{6}{|l|}{ Personality (BFI-10) } \\
\hline Extraversion & 3,28 & 1,02 & 3,33 & 1,04 & .290 \\
\hline Neuroticism & 3,06 & 0,91 & 3,21 & 0,90 & .002 \\
\hline Openness & 3,5 & 1,03 & 3,54 & 1,04 & .482 \\
\hline Conscientiousness & 4,08 & 0,75 & 4,09 & 0,74 & .936 \\
\hline Agreeableness & 3,34 & 0,81 & 3,47 & 0,78 & .004 \\
\hline
\end{tabular}

\section{Conclusions}

- The fact that still a very large number of highly distressed cancer patients refuse psycho-oncological support forces us to reconsider if the established support offers match the needs of distressed cancer patients.

Moreover we need to rethink how we communicate psycho-oncological support offers, especially for distressed men.

We have to look more closely at how previous psycho-oncological treatment and personality factors may determine the desire for psychological support.

Further multivariate analysis are necessary to examine which variables are causative for this individual and health economic problem of refusal of psychooncological support services.

Correspondig Author: Theresia Pichler, M. Sc. (theresia.pichler@mri.tum.de) 